\title{
Sequence-specific backbone resonance assignments and microsecond timescale molecular dynamics simulation of human eosinophil-derived neurotoxin
}

\author{
Donald Gagné $^{1, \dagger}$, Chitra Narayanan ${ }^{1}$, Khushboo Bafna ${ }^{2}$, Laurie-Anne Charest ${ }^{1}$, Pratul K. \\ Agarwal $^{3,4}$, and Nicolas Doucet ${ }^{1,5,6,{ }^{*}}$ \\ ${ }^{1}$ INRS-Institut Armand-Frappier, Université du Québec, 531 Boulevard des Prairies, Laval, QC, \\ H7V 1B7, Canada \\ ${ }^{2}$ Graduate School of Genome Science and Technology, University of Tennessee, Knoxville, TN, \\ 37996, United States \\ ${ }^{3}$ Computational Biology Institute and Computer Science and Mathematics Division, Oak Ridge \\ National Laboratory, 1 Bethel Valley Road, Oak Ridge, TN, 37830, United States \\ ${ }^{4}$ Department of Biochemistry, Cellular and Molecular Biology, University of Tennessee, Knoxville, \\ TN, 37996, United States \\ ${ }^{5}$ PROTEO, the Québec Network for Research on Protein Function, Engineering, and Applications, \\ 1045 Avenue de la Médecine, Université Laval, Québec, QC, G1V 0A6, Canada \\ ${ }^{6}$ GRASP, the Groupe de Recherche Axé sur la Structure des Protéines, 3649 Promenade Sir \\ William Osler, McGill University, Montréal, QC, H3G 0B1, Canada
}

\section{Abstract}

Eight active canonical members of the pancreatic-like ribonuclease A (RNase A) superfamily have been identified in human. All structural homologs share similar RNA-degrading functions, while also cumulating other various biological activities in different tissues. The functional homologs eosinophil-derived neurotoxin (EDN, or RNase 2) and eosinophil cationic protein (ECP, or RNase 3) are known to be expressed and secreted by eosinophils in response to infection, and have thus been postulated to play an important role in host defense and inflammatory response. We recently initiated the biophysical and dynamical investigation of several vertebrate RNase homologs and observed that clustering residue dynamics appear to be linked with the phylogeny and biological specificity of several members. Here we report the ${ }^{1} \mathrm{H},{ }^{13} \mathrm{C}$ and ${ }^{15} \mathrm{~N}$ backbone resonance assignments of human EDN (RNase 2) and its molecular dynamics simulation on the microsecond timescale, providing means to pursue this comparative atomic-scale functional and dynamical analysis by NMR and computation over multiple time frames.

\section{Keywords}

Ribonucleases; RNA; RNase A; EDN; RNase 2; protein orthologs; enzyme dynamics

\footnotetext{
"To whom correspondence should be addressed: Phone: (450) 687-5010, ext. 4212; Fax: (450) 686-5501; nicolas.doucet@iaf.inrs.ca. $\dagger^{\dagger}$ Present address: Structural Biology Initiative, CUNY Advanced Science Research Center, New York, NY, 10031, United States.
} 


\section{INTRODUCTION}

Eosinophil-Derived Neurotoxin (EDN), also known as human RNase 2, was first identified in the large specific granule of eosinophils (Rosenberg et al., 1989). It is the largest of the eight canonical pancreatic-like RNase superfamily members found in human, with 134 amino acids and a molecular weight of $15.5 \mathrm{kDa}$. Although EDN shares $67 \%$ identity with its closest human relative, eosinophil cationic protein (ECP, or RNase 3) (Figure 1), its ribonucleolytic activity is 50- to 100-fold higher than that of ECP (Slifman et al., 1986). In addition to degrading RNA, a number of host defense functions have been reported for this enzyme. For instance, EDN was shown to exhibit antiviral activity (Domachowske et al., 1998, Rugeles et al., 2003), in addition to being involved in the activation of dendrocytes (Yang et al., 2004), immune response modulation (Yang et al., 2004), TLR2 activation, and the increase of a Th2-type response (Yang et al., 2008). The enzyme also acts as a chemoattractant for immature human dendrocytes (Yang et al., 2003). Interestingly, its close ECP structural homolog does not share these biological activities (Ackerman et al., 1985, Young et al., 1986, Hamann et al., 1987, Lehrer et al., 1989, Hamann et al., 1990, Carreras et al., 2003, Torrent et al., 2007, Chang et al., 2010), a distinction mainly attributed to the presence of residues Arg117, Pro120, and Gln122 on loop 7 (L7, Figure 1A) (Sikriwal et al., 2012).

EDN was previously crystallized in free and ligand-bound forms (Mosimann et al., 1996, Leonidas et al., 2001, Swaminathan et al., 2002), preserving the typical catalytic triad required for ribonucleolytic activity, i.e. His15, Lys38, and His129. The enzyme also conserves the four disulfide bridges required for structural integrity, in addition to other key functional residues, such as Thr42 and Leu130 (Figure 1). As previously observed in the prototypical RNase A homolog, the catalytic His129 residue was found to adopt two distinct conformations at various $\mathrm{pH}$ values (Swaminathan et al., 2002). The ligand-bound conformation A is predominant in all homologous structures, with conformation B typically observed at lower $\mathrm{pH}$ values. EDN displays another interesting molecular distinction relative to other family members, showing a serine in the functionally important position 64 (Figure 1B) (Swaminathan et al., 2002). Much like His129, Ser64 was shown to adopt two different orientations in crystal structures. In homologous RNases, this position is normally occupied by a histidine or a lysine residue, stabilizing the negatively charged phosphate group of the RNA substrate during catalysis (Boix et al., 1999). It was hypothesized that Arg132 might play a similar role in the catalytic reaction of EDN (Sikriwal et al., 2009).

We previously observed similar conformational exchange experienced by structural homologs of the pancreatic-like RNase A superfamily (Gagné et al., 2012). This clustering millisecond timescale flexibility is concentrated within contiguous three-dimensional residue sectors of the pancreatic-like fold, and appears to be linked with the phylogeny and biological specificity of each RNase member. Similar exchange rates $\left(k_{\mathrm{ex}}\right)$ between sectors were also observed to correlate with reduced catalytic activity among selected human RNases (Gagné et al., 2012, Gagné et al., 2015b), further warranting additional investigation of functionally related RNase homologs. As a first step to better characterize this functional and NMR dynamical comparison between several members of the pancreatic-like RNase A superfamily, herein we report the ${ }^{1} \mathrm{H},{ }^{13} \mathrm{C}$ and ${ }^{15} \mathrm{~N}$ backbone resonance assignments of 
human EDN. We also describe the computational simulation of EDN on the microsecond timescale, providing an overview of its motional behavior on this particular time frame.

\section{METHODS AND EXPERIMENTS}

\section{DNA constructs, protein expression and purification}

cDNA sequence uniprot P10153 (nucleotides 28-161) was codon-optimized for Escherichia coli expression and subcloned into a NdeI/HindIII pJexpress414 (T7) cloning vector (DNA2.0, Newark, CA, USA). The ampicillin-resistant construct was transformed into $E$. coli BL21(DE3). Cells were expressed with $1 \mathrm{mM}$ IPTG in M9 minimal medium supplemented with metals, ammonium acetate, and non-essential amino acids (Invitrogen, Grand Island, NY, USA). Purification was performed as previously described (Gagné and Doucet, 2015). The protein was quantified by UV-Vis spectrophotometry using an extinction coefficient of 17,460 $\mathrm{M}^{-1} \mathrm{~cm}^{-1}$, as estimated by ExPASy ProtParam (Wilkins et al., 1999).

\section{Solution NMR experiments}

All NMR experiments were conducted at $298 \mathrm{~K}$ on samples containing $0.4-0.7$ $\mathrm{mM}{ }^{1} \mathrm{H} /{ }^{13} \mathrm{C} /{ }^{15} \mathrm{~N}$-labeled EDN and prepared in $15 \mathrm{mM}$ sodium acetate at $\mathrm{pH} 5.0$ with $10 \%$ $\mathrm{D}_{2} \mathrm{O}$. NMR experiments were carried out on a Varian (Agilent) $800 \mathrm{MHz}$ NMR spectrometer equipped with triple-resonance cold probes and pulsed field gradients. Twodimensional ${ }^{1} \mathrm{H}-{ }^{15} \mathrm{~N}$ HSQC, NOESY and three-dimensional ${ }^{1} \mathrm{H},{ }^{13} \mathrm{C},{ }^{15} \mathrm{~N} \mathrm{CCONH}$, $\mathrm{CBCA}(\mathrm{CO}) \mathrm{NH}, \mathrm{HNCACB}$ and $\mathrm{HNCO}$ spectra were collected and processed with NMRPipe. Results were further analyzed using NMRView 9.1. Sequence-specific assignments of ${ }^{1} \mathrm{H},{ }^{13} \mathrm{C}$, and ${ }^{15} \mathrm{~N}$ backbone atoms were obtained using runabout (Figure 2). A Linear Analysis of Chemical Shifts (LACS) approach was performed using NMRView 9.1 to predict the robustness of the assignments. The chemical shift $(\delta)$ of each amino acid was compared to the offset-corrected chemical shift $(\Delta \delta)$ of the BMRB entries to detect outliers, as previously described (Wang et al., 2005) (Figure 3). Residues outside three standard deviations of the mean were considered as outliers.

\section{Molecular dynamics simulations}

Molecular dynamics (MD) simulations of EDN were performed using $1 \mathrm{GQV}$ as the starting PDB structure. Pre-processing and MD simulation steps were performed using the AMBER 14 simulation package (Case et al., 2014). The protein was solvated using SPC/E water model and the system neutralized by the addition of seven $\mathrm{Cl}^{-}$ions. Simulations were performed with the AMBER FF14SB force-field at $300 \mathrm{~K}$ and NVE (constant volume and energy) conditions using GPU-enabled pmemd simulation engine for the equilibration and production runs. Equilibration was performed according to a protocol developed in our lab and described previously (Agarwal, 2004). A time-step of 2 femtoseconds (fs) was used and a production run of 500 nanoseconds (ns) was performed. As described previously (Gagné et al., 2015a), the top ten modes of the RMSFs (root mean squared fluctuations) were used to characterize the backbone $(\mathrm{Ca})$ and all-atom flexibility of simulation trajectories. A dynamic cross-correlation map (DCCM) was used to determine the correlated motions between amino acid residues of EDN. Order parameters for the NMR and MD simulations were obtained with backbone chemical shifts as input in TALOS+ (Shen et al., 2009). 


\section{RESULTS}

\section{Assignments and data deposition}

EDN backbone resonance assignments for ${ }^{1} \mathrm{H},{ }^{13} \mathrm{Ca},{ }^{13} \mathrm{C} \beta$, and ${ }^{15} \mathrm{~N}$ at $25^{\circ} \mathrm{C}$ in $15 \mathrm{mM}$ sodium acetate $\mathrm{pH} 5.0$ were acquired. The two-dimensional ${ }^{1} \mathrm{H}-{ }^{15} \mathrm{~N}$ HSQC spectrum is presented in Figure 2. It shows well-dispersed resonances, indicating that the protein is well folded. A total of 121 out of 122 non-proline ${ }^{1} \mathrm{H}^{15} \mathrm{~N}^{15}$ backbone residues $(99.2 \%)$ were assigned. Similarly, resonances were assigned for 105 out of $122 \mathrm{Ca}(86.1 \%)$ and 103 out of $120 \mathrm{C} \beta$ (85.8\%) (EDN contains two glycines, which do not have $\mathrm{C} \beta \mathrm{s}$ ). Peak positions of the assigned residues were confirmed with NOESY-HSQC and CCONH spectrum analysis. From the 122 non-proline residues, only one residue (Lys1) was unassigned, and 13 residues showed overlapping resonances (Met105-Gln116, Arg35-Tyr98, Thr24-Thr61, His73Ile108-Val125, Asn53-Asn59, and Asn92-Phe106). The ${ }^{1} \mathrm{H},{ }^{13} \mathrm{Ca},{ }^{13} \mathrm{C} \beta$, and ${ }^{15} \mathrm{~N}$ assignments have been deposited in the Biological Magnetic Resonance Data Bank (BioMagResBank, http://www.bmrb.wisc.edu) under accession number 26531.

\section{Linear Analysis of Chemical Shifts}

The Linear Analysis of Chemical Shifts (LACS) is a graphical representation of the robustness of the assignments (Wang et al., 2005). The LACS approach was used to detect inconsistencies in chemical shift referencing, representing the difference of the chemical shift for each nuclei with the offset-corrected chemical shifts corresponding with nearly 1800 BMRB entries (Figure 3). Five outliers were identified (Table S1): N-His15 (105.91 ppm, mean of $119.47 \pm 4.23 \mathrm{ppm})$, Ca-Gln21 (62.69 ppm, mean of $56.58 \pm 2.17 \mathrm{ppm})$, CaIle108 (53.39 ppm, mean of $61.59 \pm 2.77 \mathrm{ppm}), \mathrm{C} \beta$-Asn41 (44.35 ppm, mean of 38.64 $\pm 1.75 \mathrm{ppm}$ ), and HN-Asp115 (10.36 ppm, mean of $8.32 \pm 0.57 \mathrm{ppm})$.

\section{Conformational dynamics of RNase 2}

Amplitudes of motions of the backbone $\mathrm{N}-\mathrm{H}$ bond vector on the fast ps-ns timescale were probed using the square of the generalized order parameter $\left(S^{2}\right)$. Order parameters were calculated from ensemble averaged chemical shifts for the MD ensemble using TALOS+ (Shen et al., 2009). Chemical shifts for the MD ensembles were calculated from PDB coordinates using SHIFTX2 (Han et al., 2011). Order parameters obtained using the random coil index (RCI) (Berjanskii and Wishart, 2005) for the MD ensemble were nearly identical to those obtained using TALOS+ (Figure S1). Good qualitative agreement was observed between the calculated NMR and MD order parameters (Figure 4). The N-terminal region (1-40) of the protein was more rigid than the rest of the protein. Residues 65-70, 74-77, 8594 and 121-128, corresponding to loops 4, 5, 6 and 7, respectively, were observed to be most flexible on this timescale in both MD and NMR. Interestingly, the enhanced flexibility of residues 74-77 (loop 5) were also observed in EDN/ECP homologs from other organisms (unpublished data).

Conformational dynamics of RNase 2 on the microsecond ( $\mu$ s) timescale was characterized using MD simulations. Figure 5A shows the root mean square fluctuations (RMSF) calculated from the MD trajectory. The largest motions on the $\mu$ s timescale are associated with the loop regions (loops 4 and 6 ), with the $\beta$-core of the protein being mostly rigid. 
These observations are consistent with previous experimental observations of dynamical motions in homologous proteins (Gagné and Doucet, 2013). Characterization of correlated motions of RNase 2 using the dynamic cross-correlation map (Figure 5B) showed distinct regions displaying correlated (red boxes) and anti-correlated (white boxes) motions. The highest correlations and anti-correlations were observed for $\beta$-strands in the core of the protein. $\beta 3$ (residues $79-85$ ) showed correlated motions with $\beta 1$ (residues $37-45$ ) and $\beta 4$ (residues 97-104), while peripheral $\beta$-strands ( $\beta 1$ and $\beta 4$ ) showed anti-correlated motions. The dynamic loop 4 region (residues 62-71) showed anti-correlated motions with residues in the vicinity of the catalytic Lys38 (residues 33-43) and loop 6 (residues 86-92). Loop 6 residues also showed anti-correlated motions with residues near His129 (residues 129-135). Anti-correlated motions were also observed between residues adjacent to loop 6 (residues 92-98) and residues 40-47 and 10-18, both in the vicinity of catalytic residues of RNase 2, in addition to correlated motions with residues $77-83$. These observations show enhanced correlated and anti-correlated motions of residues of the dynamic loops (L4 and L6) relative to the catalytic triad residues, suggesting a potential impact on the function. Further analysis is required to characterize the role of these motions for catalytic function, both from the MD simulation and NMR relaxation perspectives.

\section{CONCLUSION}

In summary, the ${ }^{1} \mathrm{H}_{-}{ }^{13} \mathrm{C}-{ }^{15} \mathrm{~N}$ backbone resonance assignments for human EDN (RNase 2) were elucidated using a series of 2D and 3D NMR experiments. Molecular dynamics simulations on the microsecond time frame provided information on correlated and anticorrelated loop sector motions that support previous observations suggesting the conservation of dynamical motions in homologous pancreatic-like RNases (Gagné and Doucet, 2013). Dynamics on the faster timescale is observed primarily for the loop regions of EDN. The NMR resonance assignments of EDN will provide the foundation to a series of additional NMR relaxation, MD simulations, and functional experiments that will help better understand the biological role and dynamical behavior of this intriguing pancreatic-like RNase family homolog involved in human host defense mechanisms.

\section{Supplementary Material}

Refer to Web version on PubMed Central for supplementary material.

\section{Acknowledgments}

The authors thank Tara Sprules (Québec/Eastern Canada High Field NMR Facility, McGill University) and Sameer Al-Abdul-Wahid (NMR Centre, University of Guelph) for their excellent technical assistance, in addition to David Bernard (INRS-Institut Armand-Frappier) and Bruce Johnson (CUNY Advanced Science Research Center) for helpful discussions. This work was supported by the National Institute of General Medical Sciences (NIGMS) of the NIH under award number R01GM105978 (to N.D. and P.K.A.), and a Natural Sciences and Engineering Research Council of Canada (NSERC) Discovery Grant under award number RGPIN-2016-05557 (to N. D.). N.D. holds a Fonds de Recherche Québec - Santé (FRQS) Research Scholar Junior 2 Career Award. C.N. is the recipient of a postdoctoral fellowship from the Fondation Universitaire Armand-Frappier de l'INRS. D.G. and L.A.C. were respectively recipients of an NSERC Alexander Graham Bell Canada Graduate Scholarship and a M.Sc. scholarship from the Fondation Universitaire Armand-Frappier de l'INRS. 


\section{References}

Ackerman SJ, Gleich GJ, Loegering DA, Richardson BA, Butterworth AE. Comparative toxicity of purified human eosinophil granule cationic proteins for schistosomula of Schistosoma mansoni. Am J Trop Med Hyg. 1985; 34:735-45. [PubMed: 4025686]

Agarwal PK. Cis/trans isomerization in HIV-1 capsid protein catalyzed by cyclophilin A: insights from computational and theoretical studies. Proteins. 2004; 56:449-63. [PubMed: 15229879]

Armougom F, Moretti S, Poirot O, Audic S, Dumas P, Schaeli B, et al. Expresso: automatic incorporation of structural information in multiple sequence alignments using 3D-Coffee. Nucleic Acids Res. 2006; 34:W604-8. [PubMed: 16845081]

Berjanskii MV, Wishart DS. A simple method to predict protein flexibility using secondary chemical shifts. J Am Chem Soc. 2005; 127:14970-1. [PubMed: 16248604]

Boix E, Leonidas DD, Nikolovski Z, Nogues MV, Cuchillo CM, Acharya KR. Crystal structure of eosinophil cationic protein at $2.4 \AA$ resolution. Biochemistry. 1999; 38:16794-801. [PubMed: 10606511]

Carreras E, Boix E, Rosenberg HF, Cuchillo CM, Nogues MV. Both aromatic and cationic residues contribute to the membrane-lytic and bactericidal activity of eosinophil cationic protein. Biochemistry. 2003; 42:6636-44. [PubMed: 12779318]

Case D, Babin V, Berryman J, Betz R, Cai Q, Cerutti D, et al. Amber. 2014; 14

Chang KC, Lo CW, Fan TC, Chang MD, Shu CW, Chang CH, et al. TNF-alpha mediates eosinophil cationic protein-induced apoptosis in BEAS-2B cells. BMC Cell Biol. 2010; 11:6. [PubMed: 20089176]

Domachowske JB, Dyer KD, Bonville CA, Rosenberg HF. Recombinant human eosinophil-derived neurotoxin/RNase 2 functions as an effective antiviral agent against respiratory syncytial virus. $\mathrm{J}$ Infect Dis. 1998; 177:1458-64. [PubMed: 9607820]

Gagné D, Charest LA, Morin S, Kovrigin EL, Doucet N. Conservation of flexible residue clusters among structural and functional enzyme homologues. J Biol Chem. 2012; 287:44289-300. [PubMed: 23135272]

Gagné D, Doucet N. Sequence-specific backbone ${ }^{1} \mathrm{H},{ }^{13} \mathrm{C}$, and ${ }^{15} \mathrm{~N}$ resonance assignments of human ribonuclease 4. Biomol NMR Assign. 2015; 9:181-5. [PubMed: 25030111]

Gagné D, Doucet N. Structural and functional importance of local and global conformational fluctuations in the RNase A superfamily. FEBS J. 2013; 280:5596-607. [PubMed: 23763751]

Gagné D, French RL, Narayanan C, Simonovic M, Agarwal PK, Doucet N. Perturbation of the conformational dynamics of an active-site loop alters enzyme activity. Structure. 2015a; 23:225666. [PubMed: 26655472]

Gagné D, Narayanan C, Doucet N. Network of long-range concerted chemical shift displacements upon ligand binding to human angiogenin. Protein Sci. 2015b; 24:525-33. [PubMed: 25450558]

Hamann KJ, Barker RL, Loegering DA, Gleich GJ. Comparative toxicity of purified human eosinophil granule proteins for newborn larvae of Trichinella spiralis. J Parasitol. 1987; 73:523-9. [PubMed: 3598802]

Hamann KJ, Gleich GJ, Checkel JL, Loegering DA, McCall JW, Barker RL. In vitro killing of microfilariae of Brugia pahangi and Brugia malayi by eosinophil granule proteins. J Immunol. 1990; 144:3166-73. [PubMed: 2324497]

Han B, Liu Y, Ginzinger SW, Wishart DS. SHIFTX2: significantly improved protein chemical shift prediction. J Biomol NMR. 2011; 50:43-57. [PubMed: 21448735]

Lehrer RI, Szklarek D, Barton A, Ganz T, Hamann KJ, Gleich GJ. Antibacterial properties of eosinophil major basic protein and eosinophil cationic protein. J Immunol. 1989; 142:4428-34. [PubMed: 2656865]

Leonidas DD, Boix E, Prill R, Suzuki M, Turton R, Minson K, et al. Mapping the ribonucleolytic active site of eosinophil-derived neurotoxin (EDN). High resolution crystal structures of EDN complexes with adenylic nucleotide inhibitors. J Biol Chem. 2001; 276:15009-17. [PubMed: 11154698] 
Mosimann SC, Newton DL, Youle RJ, James MN. X-ray crystallographic structure of recombinant eosinophil-derived neurotoxin at $1.83 \AA$ A resolution. J Mol Biol. 1996; 260:540-52. [PubMed: 8759319]

Rosenberg HF, Tenen DG, Ackerman SJ. Molecular cloning of the human eosinophil-derived neurotoxin: a member of the ribonuclease gene family. Proc Natl Acad Sci USA. 1989; 86:4460-4. [PubMed: 2734298]

Rugeles MT, Trubey CM, Bedoya VI, Pinto LA, Oppenheim JJ, Rybak SM, et al. Ribonuclease is partly responsible for the HIV-1 inhibitory effect activated by HLA alloantigen recognition. AIDS. 2003; 17:481-6. [PubMed: 12598767]

Shen Y, Delaglio F, Cornilescu G, Bax A. TALOS+: a hybrid method for predicting protein backbone torsion angles from NMR chemical shifts. J Biomol NMR. 2009; 44:213-23. [PubMed: 19548092]

Sikriwal D, Seth D, Batra JK. Role of catalytic and non-catalytic subsite residues in ribonuclease activity of human eosinophil-derived neurotoxin. Biol Chem. 2009; 390:225-34. [PubMed: 19090717]

Sikriwal D, Seth D, Parveen S, Malik A, Broor S, Batra JK. An insertion in loop L7 of human eosinophil-derived neurotoxin is crucial for its antiviral activity. J Cell Biochem. 2012; 113:310412. [PubMed: 22581709]

Slifman NR, Loegering DA, McKean DJ, Gleich GJ. Ribonuclease activity associated with human eosinophil-derived neurotoxin and eosinophil cationic protein. J Immunol. 1986; 137:2913-7. [PubMed: 3760576]

Swaminathan GJ, Holloway DE, Veluraja K, Acharya KR. Atomic resolution (0.98 ^) structure of eosinophil-derived neurotoxin. Biochemistry. 2002; 41:3341-52. [PubMed: 11876642]

Torrent M, Cuyas E, Carreras E, Navarro S, Lopez O, de la Maza A, et al. Topography studies on the membrane interaction mechanism of the eosinophil cationic protein. Biochemistry. 2007; 46:72033. [PubMed: 17223693]

Wang L, Eghbalnia HR, Bahrami A, Markley JL. Linear analysis of carbon-13 chemical shift differences and its application to the detection and correction of errors in referencing and spin system identifications. J Biomol NMR. 2005; 32:13-22. [PubMed: 16041479]

Wilkins MR, Gasteiger E, Bairoch A, Sanchez JC, Williams KL, Appel RD, et al. Protein identification and analysis tools in the ExPASy server. Methods Mol Biol. 1999; 112:531-52. [PubMed: 10027275]

Yang D, Chen Q, Rosenberg HF, Rybak SM, Newton DL, Wang ZY, et al. Human ribonuclease A superfamily members, eosinophil-derived neurotoxin and pancreatic ribonuclease, induce dendritic cell maturation and activation. J Immunol. 2004; 173:6134-42. [PubMed: 15528350]

Yang D, Chen Q, Su SB, Zhang P, Kurosaka K, Caspi RR, et al. Eosinophil-derived neurotoxin acts as an alarmin to activate the TLR2-MyD88 signal pathway in dendritic cells and enhances Th2 immune responses. J Exp Med. 2008; 205:79-90. [PubMed: 18195069]

Yang D, Rosenberg HF, Chen Q, Dyer KD, Kurosaka K, Oppenheim JJ. Eosinophil-derived neurotoxin (EDN), an antimicrobial protein with chemotactic activities for dendritic cells. Blood. 2003; 102:3396-403. [PubMed: 12855582]

Young JD, Peterson CG, Venge P, Cohn ZA. Mechanism of membrane damage mediated by human eosinophil cationic protein. Nature. 1986; 321:613-6. [PubMed: 2423882] 


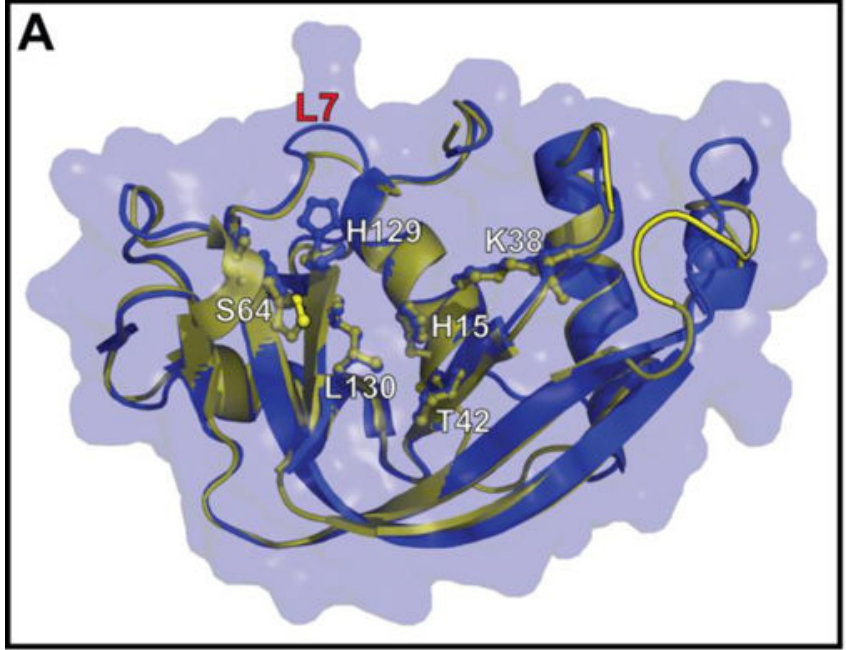

C

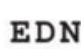

ECP

EDN
ECP

EDN

ECP

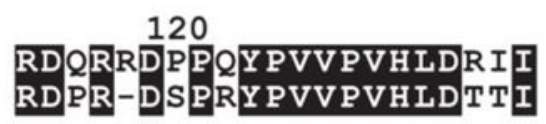

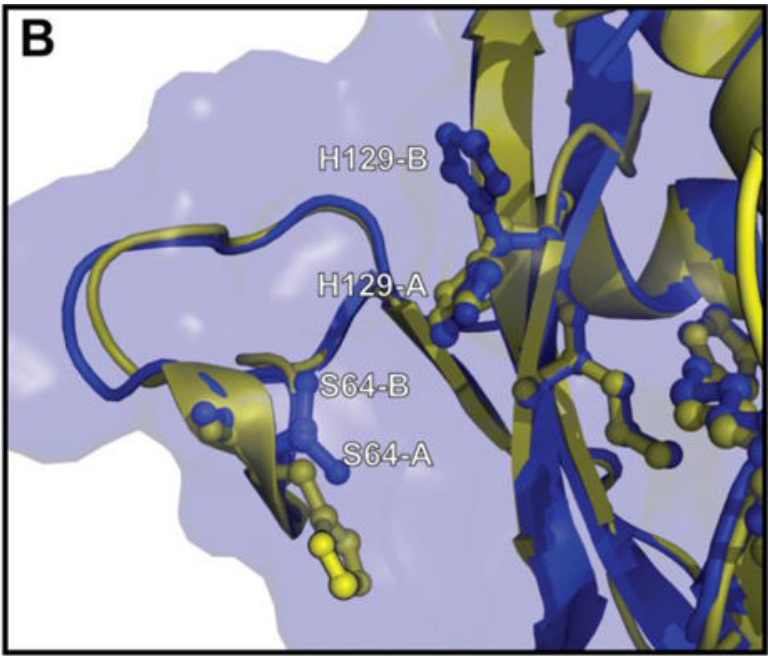

40

KPPQFTWAQWFE TQH INMT S $Q C T N A M Q V I N N Y Q R R C K N Q N T F L L T T F A N V V N V C G N$ RP PQFTRAQWFA IQH I S LN P PRCTIAMRA I NNYRWRCKNQNT FLRT T FANVVNVCGN

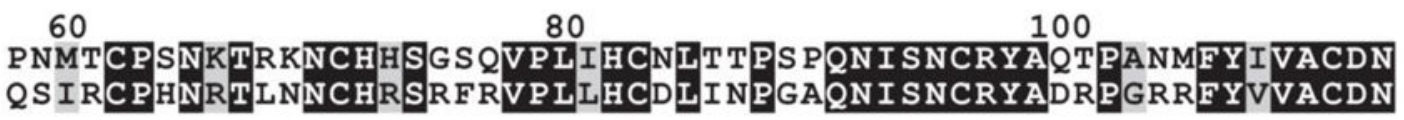

Figure 1.

Structural comparison of human eosinophil-derived neurotoxin (EDN, RNase 2) and human eosinophil cationic protein (ECP, RNase 3). (A) Structural overlay of EDN (blue, PDB 1GQV) and ECP (yellow, PDB 1QMT). Key active-site residues His15 (H15), Lys38 (K38), Thr42 (T42), Ser64 (S64), His129 (H129) and Leu130 (L130) (EDN numbering) are shown in ball-and-stick model representation, along with loop 7 (L7). (B) Magnifying view of the loop 4 environment, highlighting dual conformers for residues Ser64 and His129. (C)

Sequence comparison of EDN and ECP. Structural alignment was performed with Tcoffee Expresso (Armougom et al., 2006), and processed with ExPASy BoxShade 3.21. Sequence identity (similarity) is shown in black (gray). All 3D structures were generated with PyMOL. 


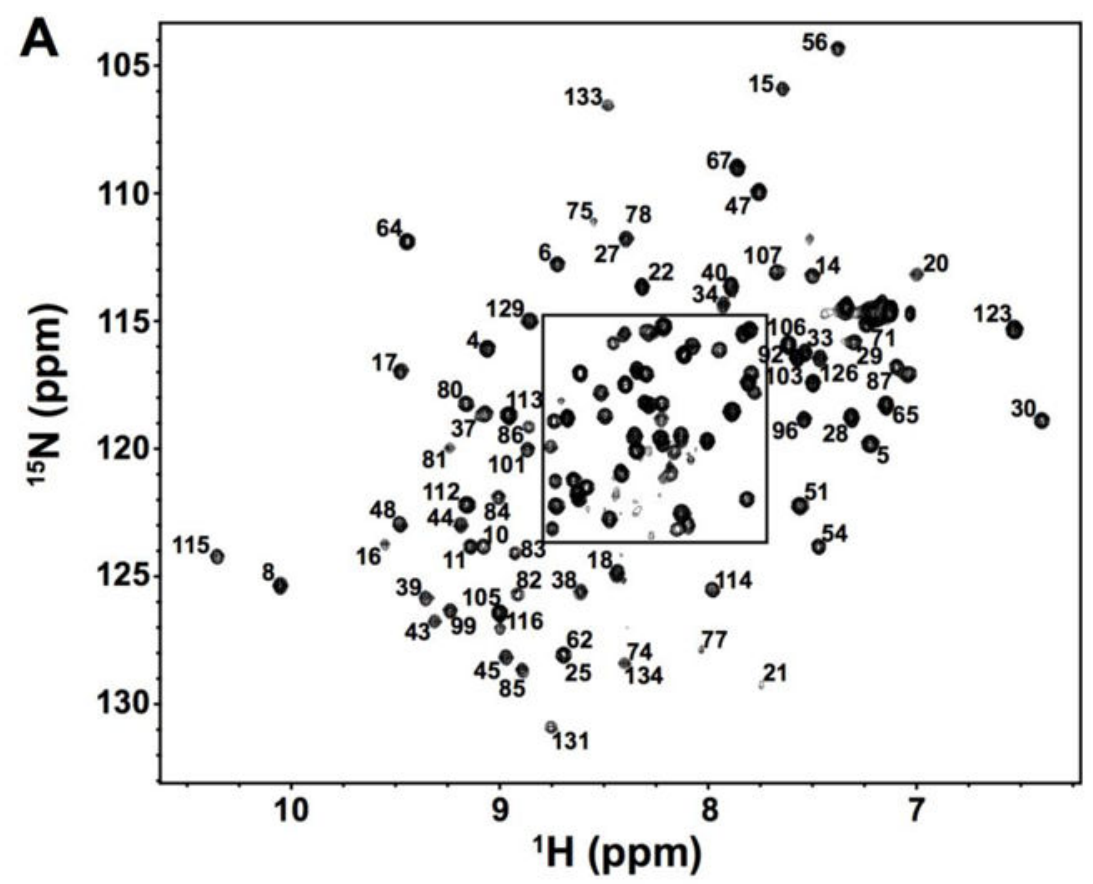

B

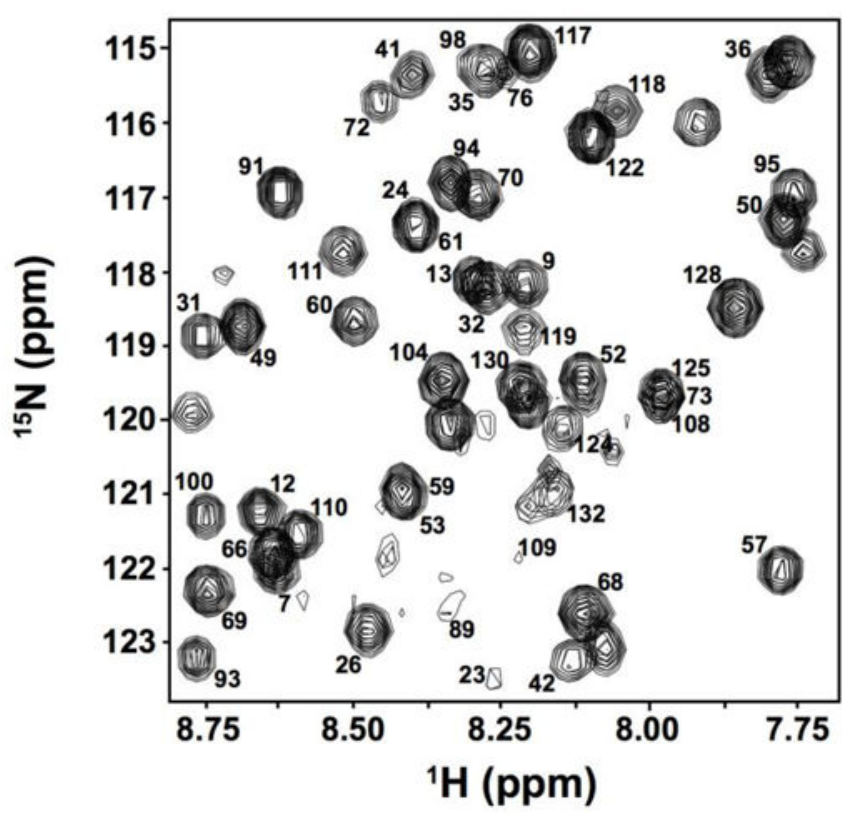

Figure 2.

Two-dimensional ${ }^{1} \mathrm{H}-{ }^{15} \mathrm{~N}$ HSQC spectrum of human eosinophil-derived neurotoxin (EDN, RNase 2) acquired in $15 \mathrm{mM}$ sodium acetate at $\mathrm{pH} 5.0\left(90 \% \mathrm{H}_{2} \mathrm{O}, 10 \% \mathrm{D}_{2} \mathrm{O}\right)$ and $298 \mathrm{~K}$. All NMR assignment experiments were acquired on a Varian Inova $800 \mathrm{MHz}$ spectrometer. Assigned resonance cross peaks are labeled with sequence specific residue numbering of EDN. Panel B is a 300X magnifying view of the central spectral region. 

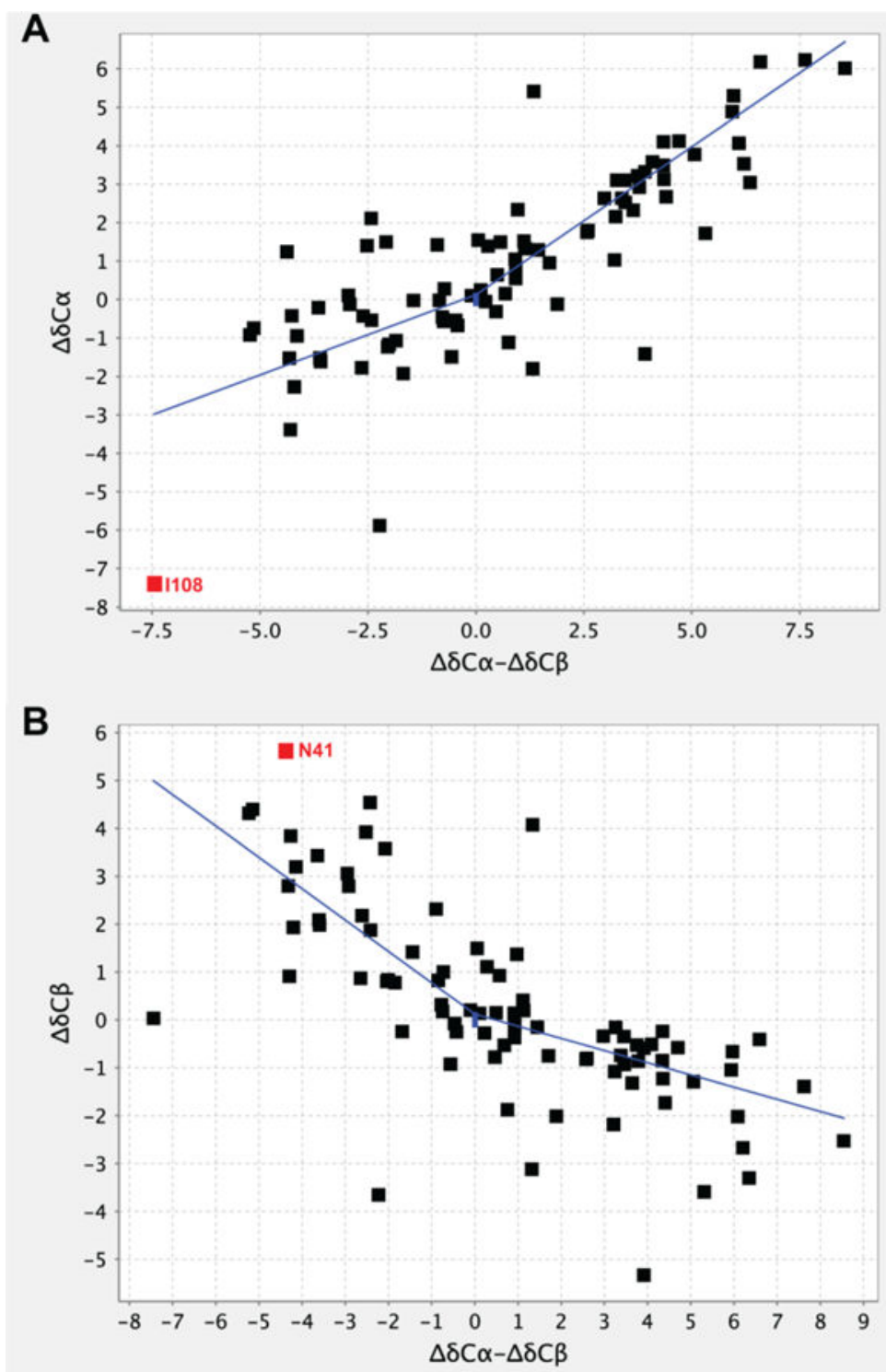

Figure 3.

Linear Analysis of Chemical Shifts (LACS) (Wang et al., 2005) for Ca and C $\beta$ resonance assignments in human EDN (RNase 2). The chemical shift $(\delta)$ of each amino acid was compared to the random coil chemical shift $(\Delta \delta)$ of a BMRB average random coil $\Delta \delta$ to produce the plots and to detect outliers. The two blue lines represent the best fitted data using lines of pre-calculated slopes. The fitted lines intersect at 0 , indicating that the data are properly referenced. $\mathrm{Ca}$ and $\mathrm{C} \beta$ outliers for Asn41 and Ile108 are labeled as red squares. The $\mathrm{Ca}-\mathrm{Gln} 21$ outlier does not appear on this figure because its $\mathrm{C} \beta$ resonance is unassigned, thus precluding calculation of the $\Delta \delta \mathrm{Ca}-\Delta \delta \mathrm{C} \beta$ parameter. 


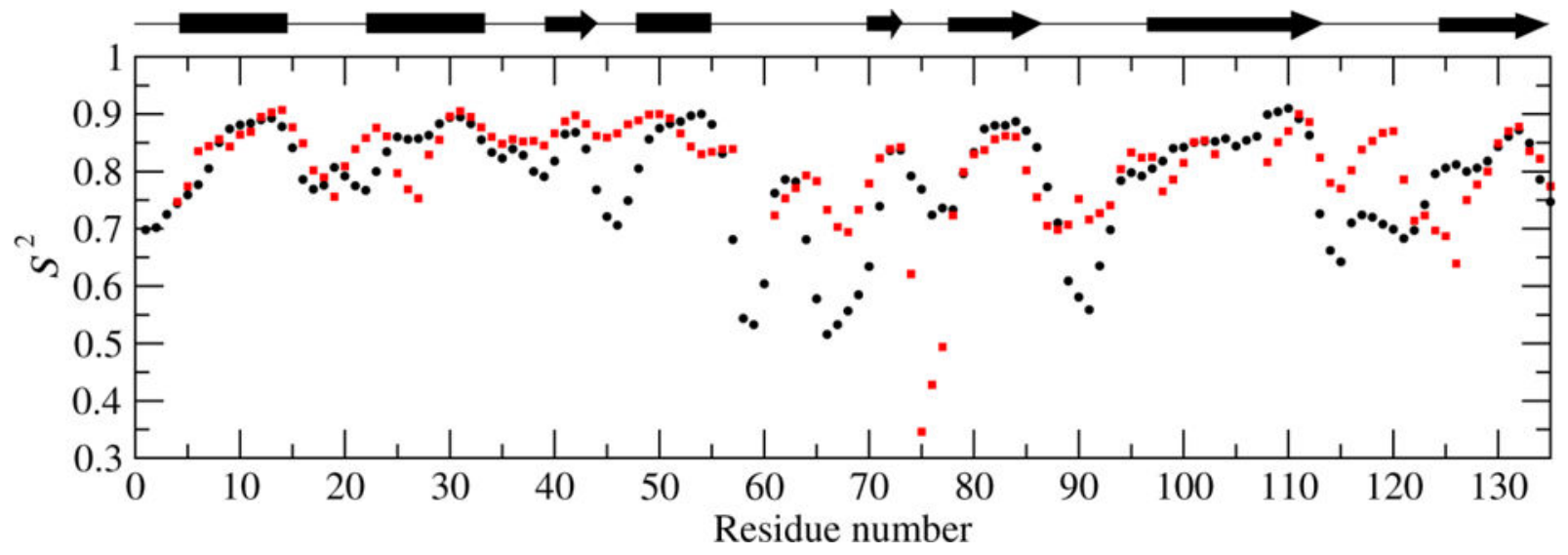

Figure 4.

Conformational dynamics of EDN (RNase 2) on the ps-ns timescale. Comparison of order parameters $\left(S^{2}\right)$ calculated for the NMR (red) and MD ensemble (black). $S^{2}$ was calculated from backbone chemical shifts using TALOS+ (Shen et al., 2009). 
A

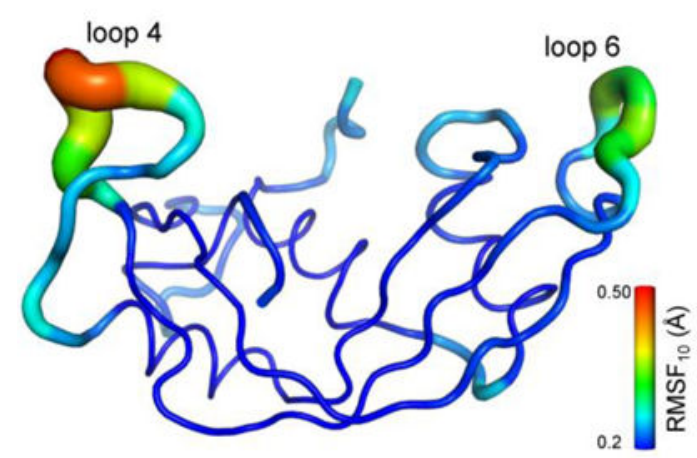

B

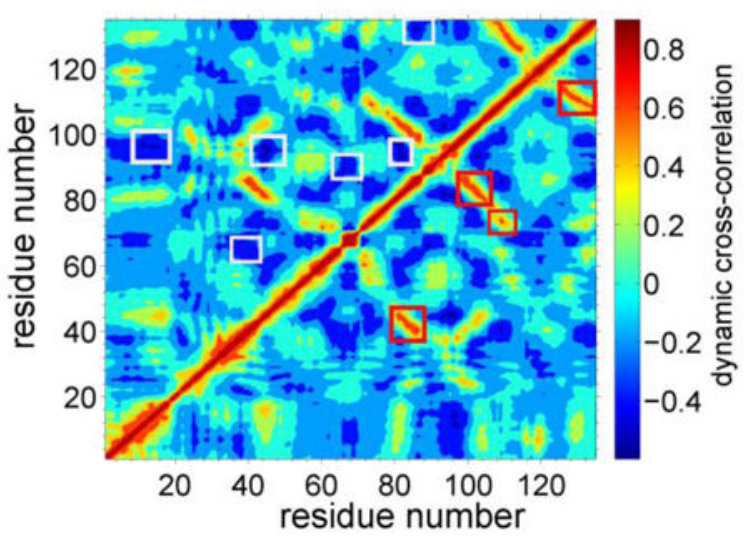

Figure 5.

Conformational dynamics of EDN (RNase 2) on the microsecond ( $\mu$ s) timescale. A) Conformational motions of EDN determined from the root mean square fluctuation (RMSF) calculated from simulation trajectories. Thickness of the tube corresponds to the flexibility of residues, with thick tubes (red end of the spectrum) corresponding to flexible regions and thin tubes (blue end of the spectrum) corresponding to less dynamic regions. B) Dynamic cross correlation map (DCCM) corresponding to the correlated motions of EDN. Values closer to 0.8 (red end of spectrum) represent correlated motions of regions within the protein, while values closer to 0.2 (blue end of the spectrum) correspond to lack of correlated motions (or anti-correlated motions). Regions showing correlated motions are identified using red boxes, while those displaying anti-correlated motions are shown using white boxes. Note that this is a symmetric plot, the anti-correlated and correlated motions are highlighted in separate sections for convenience only. 